Chapter 6

\title{
Immune Synapses Between Lymphocytes and Target Cells in Autoimmune Thyroid Diseases
}

\author{
Iwona Ben-Skowronek and Roman Ciechanek \\ Additional information is available at the end of the chapter \\ http://dx.doi.org/10.5772/53009
}

\section{Introduction}

The immune synapse is the interface between an antigen-presenting cell and a lymphocyte [1-4] as well as the interface between different lymphocytes, Natural Killer cells, and target cells [5]. This intercellular connection serves as a focal point for exocytosis and endocytosis [6]. Numerous investigations have elucidated the structure of the immunological synapse. The synapse is composed of a central region: a central supramolecular activation complex SMAC [CSMAC], a T cell receptor (TCR) cluster and associated signaling proteins, and peripheral SMAC ( pSMAC) of a ring of tight adhesion between the reacting cells [7]. The separated space of SMAC is the place of exocytic and endocytic events in this site but the precise site of signaling is not known [8]. The early signaling process occurs in peripheral microclusters in the pSMAC and the cSMAC in T and B cell synapses [9-12]. CD4 T cells form long-lived synapses with APCs - the synapses live few hours. CD8 T cells form transient synapses, lasting only minutes, because the target cells are killed $[13,14]$. In this cytotoxic synapse, activated Src kinases were detected in the CSMAC [13]. The cSMACs play an important role not only in signaling but also in receptor recycling because endosomal compartments polarize to the point immediately beneath the cSMAC of the immunological synapse [15]. The endosome comes to lie underneath the cSMAC as polarization of the microtubule skeletons occurs during synapse formation. This polarization is antigen-dependent. The receptor activation leads to accumulation of actin across the synapse and formation of an outer ring around the synapse [16,17]. The cytotoxic reaction of lymphocytes CD8 is connected with release of specialized lysosomes containing the lytic pore-forming protein perforin, which enables gransymes to lead to rapid apoptosis of the target cell [17]; the centrosome in the lymphocytes is polarized right up to the plasma membrane containing the synapse cSMAC [18]. The lytic granules are delivered to a specialized secretory domain within the synapse by moving along the microtubules toward the centro- 
some. Granule contents are then released into a small cleft between the two cells [17]. Since the overall levels of surface and endocytosed proteins remain the same regardless of ICAM-1, this suggests that ICAM-1-LFA-1 engagement in the pSMAC acts to restrict and focus endocytic and exocytic events to the center of the synapse. Griffiths suggests that the centrosome may play a role in identifying a specialized area of membrane for focal endocytosis and exocytosis [6]. An important role in formation of the immunological synapse is played by the localization of mitochondria - the mitochondria can activate and terminate the activity of immune synapses [19]. The in vivo image of T cell activation is slightly more complex. In an experimental system that uses subcutaneous injection of labeled LPS-activated dendritic cells followed by intravenous injection of naive transgenic CD8+ T cells, behavior of these cells and a three-phase model for T cell activation were observed: [20]. Phase 1 includes initial transient $\mathrm{T}$ cell-DC interactions characterized by continued rapid $\mathrm{T}$ cell migration that can last from 30 min to $8 \mathrm{~h}$ depending on the $\mathrm{pMHC}$ density. Signals in phase 1 are integrated through kinapses. Phase 2 is a period of stable T cell-DC interactions lasting $\sim 12 \mathrm{~h}$, during which cytokines such as IL-2 are produced. Signals in phase 2 are integrated through the immune synapse. Phase 3 is a return to transient $\mathrm{T}$ cell-DC interaction and rapid T cell migration during which the T cell divides multiple times and then exits the lymphoid tissue. The correct interpretation of these stop and go signals is critical for generation of effector and memory T cells [21,22].

The aim of the study is presentation of the ultrastructure of immune synapses between T- cells and plasma cells and target cells in vivo in autoimmune thyroid diseases.

\section{Material and methods}

\subsection{Patients}

A group of children and adolescents was chosen for the study to exclude the impact of aging processes and other diseases connected with age: circulatory disorders, arterial sclerosis, and drug use.

The study involved 90 children: 30 children affected with Graves' disease, 30 children with Hashimoto's thyroiditis and 30 children as a control group. The children were treated in the Department of Pediatric Endocrinology and Neurology in Lublin and in the Pediatric Department in Rzeszow in the years 1994 - 2007 and operated on in the Surgery Department of the Regional Hospital in Lublin and in the Regional Hospital in Rzeszow.

The investigation was accepted by the local Ethical Committee at the Medical University in Lublin.

\subsubsection{Control group}

The control group consisted of 30 children aged 6-19 who had died in accidents and of other non-autoimmune diseases; the thyroid specimens were taken during autopsy $(n=25)$. Some specimens were taken during a surgical operation of thyroglossal cysts and during surgery of parathyroid glands $(n=5)$. These were fragments of routinely sampled tissue specimens for 
standard pathologic investigations. All the children were in euthyreosis [Tab.1]. All children's parents signed an informed consent before autopsy or surgical operation.

\subsubsection{Patient qualification procedure}

All patients' parents signed an informed consent before these investigations.

All the patients received physical examination to assess the goiter and clinical signs and symptoms of thyroid disorders. The TSH (Thyroid-stimulating hormone), fT4 (free thyroxin) and TT3 (total triiodothyronine) hormones were assayed by MEIA (Abbott Kit, Langford, Ireland). The levels of TSH receptor antibodies were measured by RIA (TRAK assay BRAHMS Diagnostica GmbH, Berlin, Germany). The thyroperoxidase (TPO) and thyroglobulin (TG) antibodies were assayed by LIA (Lumitest BRAHMS Diagnostica GmbH, Berlin, Germany).

In the patients with Graves' disease, symptoms of thyrotoxicosis: goiter, tachycardia, sleeplessness, anxiety, high diastolic/systolic blood pressure amplitude, an increase in fT4 (mean $3.8 \pm 0.7 \mathrm{ng} / \mathrm{dl}$ ) and TT3 (mean $363 \pm 175.3 \mathrm{ng} / \mathrm{dl}$ ), and a decrease in TSH (mean $0.004 \pm 0.003 \mathrm{mU} /$ 1) were observed. The levels of antibodies against the TSH receptor (TRAB) $(7-462 \mathrm{U} / \mathrm{ml})$ and the levels of TPO antibodies (21-6663U/ml) and TG antibodies $(25-13351 \mathrm{U} / \mathrm{ml})$ were usually increased. The patients were treated with methimazole in initial doses $0.9-0.5 \mathrm{mg} / \mathrm{kg}$ b.w./day during 4-6 weeks and after that time, when in euthyreosis, they got maintenance doses c.a.0.1 $\mathrm{mg} / \mathrm{kg}$ b.w./ day (mainly $5 \mathrm{mg} /$ day) in combination with a low dose of l-thyroxin $(25 \mu \mathrm{g} /$ day) during 18-24 months. Children with Graves' disease, whose early relapses of hyperthyreosis necessitated surgery treatment - thyroidectomy after 18-36 months, were qualified for the investigation [Tab. 1].

Hashimoto's thyroiditis was recognized in patients with parenchymal or nodular large size goiter accompanied by pressure to other neck structures in the phase of euthyreosis or hypothyreosis, rarely in hyperthyreosis (Hashitoxicosis). In ultrasonography, a non-homogenous structure of the thyroid was observed. The levels of TPO Ab and TG Ab were increased, but the levels of the TRAb were in normal ranges. In histopathological examination, mononuclear lymphatic infiltrations in the thyroid parenchyma were detected, and Hashimoto's thyroiditis was diagnosed. Before surgery, the patients were usually treated with 1-thyroxin 25-100 $\mu$ g/day. [Tab. 1].

\begin{tabular}{cccccccc}
\hline & $\begin{array}{c}\text { Patients } \\
\text { number }\end{array}$ & $\begin{array}{c}\text { Age } \\
\text { [years] }\end{array}$ & $\begin{array}{c}\text { TSH } \\
\mathbf{m I U / L}\end{array}$ & $\begin{array}{c}\mathbf{f T 4} \\
\mathbf{n g} / \mathbf{d l}\end{array}$ & $\begin{array}{c}\text { TPO Ab } \\
\text { IU/L }\end{array}$ & $\begin{array}{c}\text { TG Ab } \\
\text { IU/L }\end{array}$ & $\begin{array}{c}\text { TSI } \\
\text { IU/L }\end{array}$ \\
\hline Graves' disease & 30 & $5-19$ & $0,001-0,005$ & $3,3-5,1$ & $21-6663$ & $25-13351$ & $7-462$ \\
\hline $\begin{array}{c}\text { Hashimoto's } \\
\text { thyroiditis }\end{array}$ & 30 & $8-19$ & $0,600-98,800$ & $0,1-2,3$ & $132-9856$ & $128-14567$ & $0-0,99$ \\
\hline $\begin{array}{c}\text { Control group } \\
\text { Normal ranges }\end{array}$ & 30 & $6-19$ & $0,270-4,200$ & $\times$ & $x$ & $x$ & $\times$ \\
\hline
\end{tabular}

Table 1. Characteristics of the patients examined before treatment 


\section{Ultrastructural investigations}

Specimens for ultrastructural investigations were obtained during thyroidectomy. Small segments of thyroid were cut into $0,5 \mathrm{~mm}^{3}$ pieces and fixed in $4 \%$ glutaraldehyde in $0,1 \mathrm{M}$ cacodylate buffer, $\mathrm{pH} 7.4$ for $24 \mathrm{~h}$ in $4^{\circ} \mathrm{C}$, post fixed in $2 \%$ OsO 4 in the same buffer for $1 \mathrm{~h}$ in room temperature, dehydrated in a graded series (up to $100 \%$ ) of ethanol and embedded in 812 Epon. They were then polymerized at $60^{\circ} \mathrm{C}$. Five specimens were taken from every thyroid from each patient with Graves' disease, Hashimoto's thyroiditis, and from the control group. Epon blocks were cut with an RMC MT-7 ultramicrotome, USA. From every specimen were analyzed serial 10 slides. Ultrathin sections were contrasted with uranyl acetate and lead citrate and examined under the EM 900 Zeiss Germany Electron Microscope.

\section{Results}

In the control group of children without a thyroid disease, lymphocytes in the interstitium of the thyroid gland were observed sporadically. The lymphatic cells did not cross the basal membrane of thyroid follicles, were not in contact with thyrocytes and did not form groups. In Graves' disease, T cells that crossed the basal membrane of the vesicles were observed to be in contact with thyrocytes. The lymphocytes migrated to thyroid follicles from capillary vessels or from lymphatic follicles. The migrating T-cells had numerous projections - lamellipodia on their surface. Polarization of cell organelles was already visible in narrow capillaries. The lamellipodia, mitochondria, and the Golgi system were located in the same part of the lymphocyte [Fig.1]. T cells, which penetrated across the basal membrane between thyrocytes, looked similar [Fig. 2]. The T- cells formed numerous junctions with thyrocytes. The structure of these connections was similar to zonula occludens with an area of cell membrane fusion and area of free spaces between cells, in which protein substances were secreted [Fig.2]. The T-cells were not polarized in those connections with thyrocytes. The thyrocytes were not damaged, but were active and had numerous mitochondria, secretory vesicles and a big amount of euchromatin in the nuclei [Fig. 2].

In Hashimoto's thyroiditis, the sites of contact between T-cells and lymphocytes had the character of an immune synapse, too. The synapse, however, looked different. The T-cells were polarized - the centrioles, mitochondria, Golgi system, and secretory vesicles were present in the part connected with the thyrocytes. The synapse was composed of a distal part - an adhesion zone, and a central part - a space in which electron dense substances were secreted [Fig.3]. The thyrocytes staying in contact with T-cells exhibited the features of apoptosis: dark, concentrated heterochromatin in the nucleus, swollen mitochondria, and enlarged cisterns of endoplasmic reticulum.

In AITD, synapses between plasma cells and thyrocytes were observed. In Graves' disease, synapses were formed in the distal part - zonula adherens - without fusion of thyrocyte and plasmocyte cell membranes and in the central part - the space between membrane of plasma cells and thyrocytes. Electron dense substances from the rough endoplasmic reticulum of the 


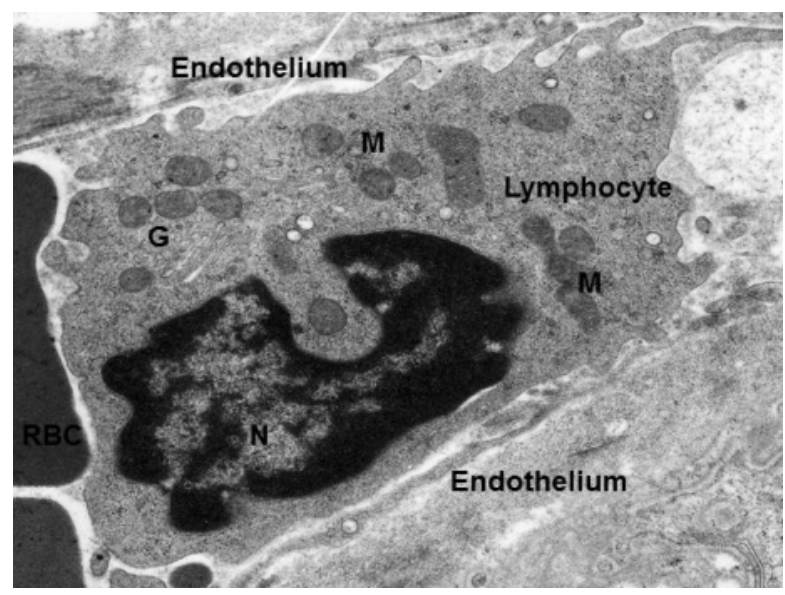

Figure 1. The lymphocyte migrating in the capillary vessel. Polarization of the lymphatic cell is visible: lamellipodia, mitochondria, and Golgi complex are present on the same side. N- nucleus, M- mitochondria, G-Golgi system, RBC-red blood cell. TEM magn. 15 000x

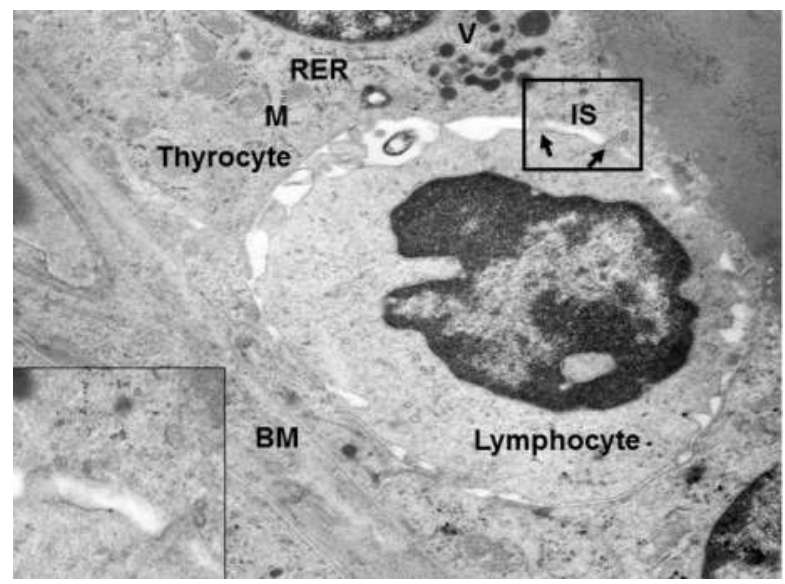

Figure 2. The lymphocyte between thyrocytes in the thyroid follicle in Graves' disease. The lymphocyte formed numerous immune synapses with the thyrocyte. The immune synapses are limited by zonula occludens (pSMAC with fusion of cell membranes). The space (cSMAC) is visible in the center of the immune synapse. The thyrocytes are active without signs of damage. RER - rough endoplasmic reticulum, M-mitochondria, V-secretory vesicle, MB- basal membrane, IS- immune synapse. TEM magn. 15 000x.

plasma cells - most probably immunoglobulins - were secreted to this space [Fig.4]. Immunoglobulins encrusted the basal membrane of thyrocytes. The thyroid cells staying in contact with plasma cells were active: with a big amount of euchromatin in the nucleus, numerous secretory vesicles, and abundant microvilli [Fig.4]. 


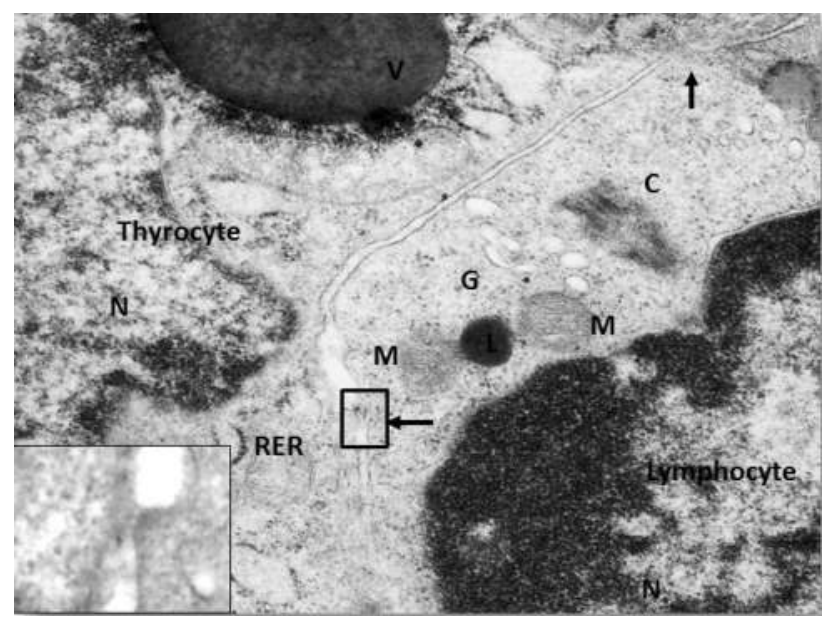

Figure 3. The lymphocyte between thyroid follicles in Hashimoto's thyroiditis. The lymphocyte is connected with the thyrocyte by an immune synapse limited by zonula adherens without cell membrane fusion (pSMAC). The space (CSMAC) is visible in the center of the immune synapse. The lymphocyte organelles are polarized under the synapse: centriole, mitochondria, granules, and Golgi complex. Signs of damage are present in the thyrocyte: enlarged cisterns of rough endoplasmic reticulum. C-centriole, M-mitochondria, G-Golgi system, L-lysosome, RER-rough endoplasmic reticulum, $\mathrm{N}$-nucleus. TEM magm.25000x

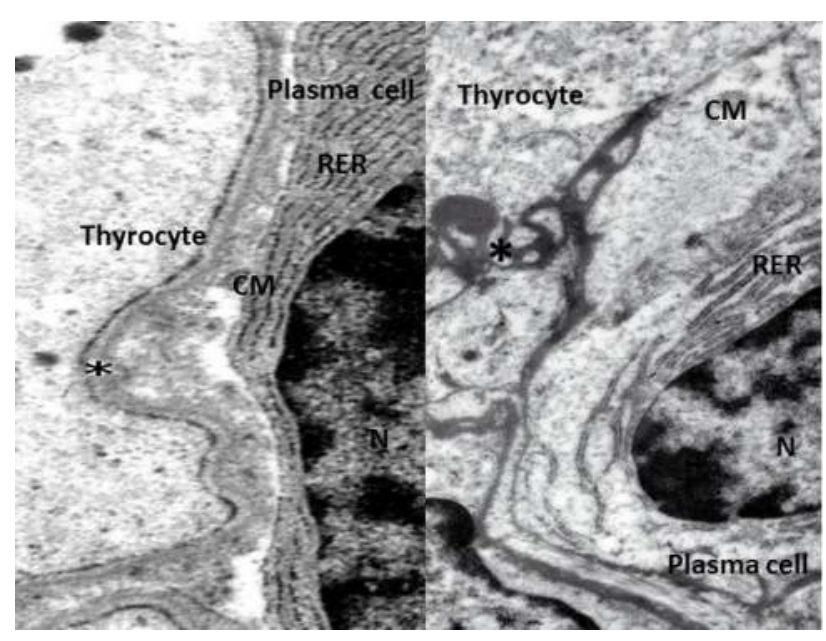

Figure 4. The plasma cells in contact with thyrocytes in Graves' disease. a) The space of the immune synapse in which electron dense substances, probably antibodies, are secreted. Enlarged cisterns of endoplasmic reticulum were observed in the thyrocyte. RER-rough endoplasmic reticulum, CM cell membrane, * immunoglobulins. TEM magn. 25 000x. b)The advanced phase of the immune reaction: the deposits of immunoglobulins in the space between the thyrocyte and plasmocyte. The space of the immune synapse is limited by zonula adherens. CM - cell membrane, RER rough endoplasmic reticulum, * immunoglobulins deposit. TEM magn. $25000 x$ 
In Hashimoto's thyroiditis, polarization of plasma cells was observed; the centrioles and Golgi system, mitochondria and well-developed rough endoplasmic reticulum were observed in the part connected with thyrocytes. In some areas of the contact places, secretion of substances with medium electron density from plasma cells to thyrocytes was observed [Fig.5]. The plasma cells adhered in a large area to thyrocytes, and the thyrocytes exhibited features of damage and destruction: fragmentation of the endoplasmic reticulum, edema of mitochondria, and condensation of the chromatin in the nucleus. In advanced stages, destruction and fragmentation of thyrocytes were observed.

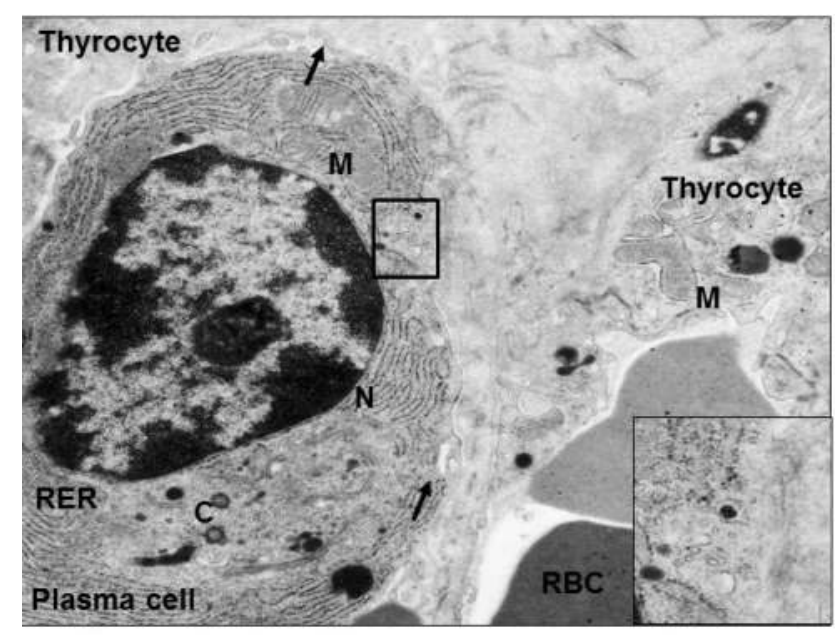

Figure 5. The plasma cell in contact with thyrocyte in Hashimoto's thyroiditis. The immune synapse is composed of a surrounding adhesion zone (pSMAC) in the central part of the site of exocytosis of electron dense substances and vesicles from plasma cell. Polarization of the organelle is visible in the plasma cell: centrioles, lysosomes, and mitochondria in the region of immune synapse. The thyrocyte is damaged with edema of mitochondria and destruction of endoplasmic reticulum. C-centriole, M-mitochondrium, RER- rough endoplasmic reticulum, N-nucleus. TEM magm.25 000x

\section{Discussion}

The immune synapses occurring in the thyroids of patients with Graves' disease were similar to the synapse described by Dustin [23]. Dynamic studies with planar bilayers further showed that the immune synapse was formed through a nascent intermediate in which activating TCR clusters are formed first in the PSMAC and then move to the cSMAC region in an F-actindependent process in a few minutes to form the pattern [7].

In the connection between thyrocytes and T-cells, the zonula occludens in Graves' disease and zonula adherens in Hashimoto's thyroiditis seem to be the peripheral pSMAC and the space in the center can correspond to the central supramolecular activation complex cSMAC. 
An interesting observation is the difference in polarization of lymphocytes in immune synapses. The lymphocyte stimulating thyrocytes in Graves' disease were not polarized, but the cytotoxic lymphocytes in Hashimoto's thyroiditis had polarized organelles in the cytoplasm.

The polarization of the T-cell, i.e. formation of a center with a centriole, mitochondria, and Golgi complex suggested special organization of the cellular tubules and filaments. Actin filaments (F-actin) play a critical role throughout the various stages of $\mathrm{T}$ cell activation. In the steady state, actin polymerization at the leading edge and cytoskeletal contraction at the lamelliopodium mediate rapid migration [24].The microtubule organizing center (MTOC) and microtubule network of the cell provide a molecular way for vesicle movement and structural support for polarized cell functions. Within seconds after TCR stimulation, the MTOC mobilizes and polarizes to the immune synapse in T cells. Polarization is important for efficient trafficking and directed secretion of cytolytic granules and cytokines for secretion at the synapse [25-27]

Previous studies [28-30] report that mitochondria accumulate at the immunological synapse following T-cell stimulation. The fusion factor DRP1 (dynamin-related protein 1) regulates mitochondria positioning close to the peripheral supramolecular activation cluster (pSMAC), which together with the central SMAC forms the immune synapse in T cells [19]. The immune synapse controls calcium signals and calcium-dependent T-cell functions [31]. Our observations in vivo are similar to pictures from the electron microscope from investigations in cell culture published by Tsun [32].

Probably, the polarization of organelles in the cytotoxic lymphocytes observed is connected with transport of cytotoxic substances from these cells to thyrocytes.

Stinchcombe [33] observed NK cells conjugated with B-cells with glycolipid-pulsed CD1bearing targets. High-resolution electron micrographs of the immunological synapse formed between NK and iNKT cytolytic cells with their targets revealed that, in both NK and iNKT cells, the centrioles could be found associated (or 'docked') with the plasma membrane within the immunological synapse. Secretory clefts were visible within the synapses formed by both NK and iNKT cells, and secretory lysosomes were polarized along microtubules leading towards the docked centrosome. The Golgi apparatus and recycling endosomes were also polarized towards the centrosome at the plasma membrane within the synapse [33]. It seems that the polarization process is connected with the cytotoxic interactions between T-cells and thyrocytes in Hashimoto's thyroiditis.

The immune synapse between plasma cells and APCs has been seldom described. Batista described the immunological synapse between plasma cells and antigen presenting cells [34]. We observed two types of immune synapses between plasma cells and thyrocytes in AITD. In Graves' disease, they are the stimulating synapses: immunoglobulins encrusting the basal membrane of the thyrocyte were secreted in the central space of the synapse and were probably connected with TSH- receptors. In the last phase of this process, deposits of immunoglobulins were visible. The similar change were observed in kidney $[35,36]$. In glomerulonephritis, subendothelial complement deposits [36,37] and sun epithelial (similar to situation in thyroid) 
immunoglobulins deposits $[35,36]$ were observed. In Hashimoto's thyroiditis, the plasma cells were polarized and formed the microtubule organizing center (MTOC) consisting of centrioles, mitochondria and Golgi apparatus and probably microtubules and microfilaments. The peripheral zonula adherens surrounded the place of immunoglobulin secretion, but the immunoglobulins penetrated to thyrocytes and probably led to damage to these cells [38].

\section{Conclusions}

- Immune synapses between T-cells and plasma cells with thyroid's epithelial cells were found in AITD.

- In the ultrastructure of the synapse, peripheral zonula occludens or zonula adherens and a central space were observed in all types of the immune synapses.

- The lymphocytes forming the cytotoxic synapse were characterized by presence of a microtubule-organizing center.

\section{Acknowledgements}

This work was supported by Grant 2P05E04327 from the Polish Ministry of Science and Higher Education.

\section{Author details}

Iwona Ben-Skowronek ${ }^{1}$ and Roman Ciechanek ${ }^{2}$

*Address all correspondence to: skowroneki@interia.pl

*Address all correspondence to: ciechanek.r@wp.pl

1 Department of Paediatric Endocrinology and Diabetology, Medical University in Lublin, Poland

2 Division of Surgery, Provincial Specialist Hospital in Lublin,, Poland

Competing interests. The authors declare that they have no competing interests.

Ethics. The investigation was accepted by the local Ethical Committee at the Medical University in Lublin. All children's parents signed an informed consent before autopsy or surgical operation. 


\section{References}

[1] Poo WJ, Conrad L, Janeway Jr. CA. Receptor-directed focusing of lymphokine release by helper T cells. Nature.1988. 332:378-380.

[2] Kupfer A, Swain SL, Singer SJ. The specific direct interaction of helper T cells and antigen-presenting B cells. II. Reorientation of the microtubule organizing center and reorganization of the membrane-associated cytoskeleton inside the bound helper $\mathrm{T}$ cells. J. Exp. Med.1987. 165:1565-1580.

[3] Kupfer A, Singer SJ. The specific interaction of helper T cells and antigen-presenting B cells. IV. Membrane and cytoskeletal reorganizations in the bound T cell as a function of antigen dose. J. Exp. Med.1989. 170:1697-1713. doi:10.1084/jem.170.5.1697.

[4] Grakoui A, Bromley SK, Sumen C, Davis MM., Shaw AS, Allen PM, Dustin ML. The immunological synapse: a molecular machine controlling $\mathrm{T}$ cell activation. Science 1999, 285 (5425): 221-227.

[5] Davis DM, Chiu I, Fassett M, Cohen GB, Mandelboim O, Strominger,JL. The human natural killer cell immune synapse. Proc Natl Acad Sci U S A 1999, 96 (26): 15062-7.

[6] Griffiths GM, Tsun,A, Stinchcombe JC. The immunological synapse: a focal point for endocytosis and exocytosis. J Cell Biol. 2010 (189) 3:390-408.

[7] Monks CR, Freiberg BA, Kupfer H., Sciaky N, Kupfer A. Three-dimensional segregation of supramolecular activation clusters in T cells. Nature 1998, 395:82-86.

[8] Dustin ML. The cellular context of T cell signaling. Immunity 2009, 30:482-492.

[9] Campi G, Varma R, Dustin ML. Actin and agonist MHC-peptide complex-dependent T cell receptor microclusters as scaffolds for signaling. J. Exp. Med. 2005,202:1031-1036.

[10] Yokosuka T, Sakata-Sogawa K, Kobayashi W, Hiroshima M, Hashimoto-Tane A, Tokunaga M, Dustin ML, Saito T. Newly generated T cell receptor microclusters initiate and sustain T cell activation by recruitment of Zap70 and SLP-76. Nat. Immunol.2005, 6:1253-1262.

[11] Varma R, Campi G, Yokosuka T, Saito T, Dustin ML. T cell receptor-proximal signals are sustained in peripheral microclusters and terminated in the central supramolecular activation cluster. Immunity 2006, 25:117-127.

[12] Depoil D, Fleire S, Treanor BL, Weber M, Harwood NE, Marchbank KL, Tybulewicz VL, Batista FD. CD19 is essential for B cell activation by promoting B cell receptorantigen microcluster formation in response to membrane-bound ligand. Nat. Immunol 2008, 9:63-72.

[13] Beal AM, Anikeeva N, Varma R, Cameron TO, Vasiliver-Shamis G, Norris PJ, Dustin ML, Sykulev Y. Kinetics of early T cell receptor signaling regulate the pathway of lytic granule delivery to the secretory domain. Immunity 2009. 31:632-642. 
[14] Jenkins MR, Tsun A, Stinchcombe JC, Griffiths GM. The strength of T cell receptor signal controls the polarization of cytotoxic machinery to the immunological synapse. Immunity. 2009.31:621-631.

[15] Das V, Nal B, Dujeancourt A, Thoulouze MI, Galli T, Roux P, Dautry-Varsat A, Alcover A. Activation-induced polarized recycling targets $\mathrm{T}$ cell antigen receptors to the immunological synapse; involvement of SNARE complexes. Immunity. 2004. 20:577588.

[16] Stinchcombe JC, Barral DC, Mules EH, Booth S, Hume AN, Machesky LM, Seabra MC, Griffiths GM. Rab27a is required for regulated secretion in cytotoxic T lymphocytes. J. Cell Biol.2001. 152:825-834.

[17] Stinchcombe JC, Griffiths GM. Secretory mechanisms in cell-mediated cytotoxicity. Annu. Rev. Cell Dev. Biol.2007. 23:495-517.

[18] Stinchcombe JC, Majorovits E, Bossi G, Fuller S, Griffiths GM. Centrosome polarization delivers secretory granules to the immunological synapse. Nature. 2006.443:462-465.

[19] Junker C, Hoth M. Immune synapses: mitochondrial morphology matters. EMBO Journal 2011, 30:1187-1189.

[20] Mempel TR, Henrickson SE, Von Andrian UH. T-cell priming by dendritic cells in lymph nodes occurs in three distinct phases. Nature. 2004;427:154-59.

[21] Hosseini BH, Louban I, Djandji D, Wabnitz GH, Deeg J, Bulbuc N, et al. Immune synapse formation determines interaction forces between $\mathrm{T}$ cells and antigen-presenting cells measured by atomic force microscopy. Proc Natl Acad Sci USA. 2009;106:17852-17857.

[22] Scholer A, Hugues S, Boissonnas A, Fetler L, Amigorena S. Intercellular adhesion molecule-1-dependent stable interactions between $\mathrm{T}$ cells and dendritic cells determine CD8+ T cell memory. Immunity. 2008;28:258-70.

[23] Dustin ML. The immunological synapse. Arthritis Res Therapy. 2002. 4 (Suppl 3): 119-125.

[24] Giannone G, Dubin-Thaler BJ, Rossier O, Cai Y, Chaga O, et al. Lamellipodial actin mechanically links myosin activity with adhesion-site formation. Cell. 2007;128:56175.

[25] Geiger B, Rosen D, Berke G. Spatial relationships of microtubule-organizing centers and the contact area of cytotoxic T lymphocytes and target cells. J Cell Biol. 1982;95:13743.

[26] Kupfer A, Dennert G, Singer SJ. Polarization of the Golgi apparatus and the microtubule-organizing center within cloned natural killer cells bound to their targets. Proc Natl Acad Sci USA. 1983;80:7224-28.

[27] Kupfer A, Mosmann TR, Kupfer H. Polarized expression of cytokines in cell conjugates of helper T cells and splenic B cells. Proc Natl Acad Sci USA. 1991;88:775-79. 
[28] Quintana A, Schwindling C, Wenning AS, Becherer U, Rettig J, Schwarz EC, Hoth M. $\mathrm{T}$ cell activation requires mitochondrial translocation to the immunological synapse. Proc Natl Acad Sci USA. 2007. 104: 14418-14423.

[29] Contento RL, Campello S, Trovato AE, Magrini E, Anselmi F, Viola A. Adhesion shapes T cells for prompt and sustained T-cell receptor signaling. EMBO J 2010.29: 4035-4047.

[30] Baixauli F, Martín-Cófreces NB, Morlino G, Carrasco YR, Calabia-Linares C, Veiga E, Serrador JM, Sánchez-Madrid F. The mitochondrial fission factor dynamin-related protein 1 modulates T-cell receptor signaling at the immune synapse. EMBO J 2011.30: 1238-1250.

[31] Kummerow C, Junker C, Kruse K, Rieger H, Quintana A, Hoth M The immunological synapse controls local and global calcium signals in T lymphocytes. Immunol Rev . 2009.231: 132-147.

[32] Tsun A, Qureshi I, Stinchcombe JC, Jenkins JR, de la Roche M, Kleczkowska J, Zamoyska R, Griffiths GM. Centrosome docking at the immunological synapse is controlled by Lck signaling. J. Cell Biol.2011;192:663-674, (10.1083/jcb.201008140).

[33] Stinchcombe JC, Salio M, Cerundala V, Pende D, Arico M, Griffiths GM. Centriole polarization to the immunological synapse direct secretion from cytolytic cells of both the innate and adaptive immune systems. BMC Biology 2011, 9-45.

[34] Batista, F.D., D. Iber, M.S. Neuberger. B cells acquire antigen from target cells after synapse formation. Nature.2001. 411:489-494.

[35] Qiu Y, Korteweg C, Chen Z, Li J, Luo J, Huang G and Gu J. Immunoglobulin G expression and its colocalization with complement proteins in papillary thyroid cancer. Modern Pathology 2012; 25, 36-45

[36] Howie AJ. Handbook of Renal Biopsy Pathology. Springer $2^{\text {nd }}$ edition 2008:175-182.

[37] Licht C, Hoppe B. Complement defects in children which result in kidney diseases: diagnosis and therapy. In Complement and kidney disease. Edited by Zipfel PF. BaselBoston-Berlin 2006 Bikhauser Verlag: 184-197.

[38] Rebuffat SA, Nguyen B,Robert B, Castex F, Peraldi Roux S. Antiperoxidase AntibodyDependent Cytotoxicity in Autoimmune Thyroid Disease. J Clin Endocrinol Metab, 2008;93;3: 929-934 